\title{
Slow light by means of coherent population effects
}

\section{Giovanni Piredda}

Robert W. Boyd
The Institute of Optics, University of Rochester, Rochester, New York 14627

The Institute of Optics, University of Rochester, Rochester, New York 14627

Several groups have recently reported the observation of slow light propagation speeds based on the process of coherent population oscillations (CPO) under conditions such that the laser line width exceeds the width of the CPO transparency window. We explain this apparently paradoxical result by noting that the CPO effect is sensitive only to intensity variations of the incident laser field that occur on time scales comparable to or shorter than the population relaxation time of the material medium. Laser linewidths are associated primarily with phase fluctuations or with intensity fluctuations on much shorter time scales. [D0I: 10.2971/je0s.2007.07004]

Keywords: Slow light, coherent population oscillations, non-collinear propagation

\section{I n t rod uct io n}

There has recently been a flurry of interest in slow- and fastlight methods, inspired both by the conceptual intrigue of the possibility of exercising great control over the propagation velocity of pulses through material systems [1] and by the potential applications of these techniques in the fields of photonics and optical switching. Extreme values of pulse velocities $(v<\langle c, v\rangle c$, and $v$ negative) have been reported [2]-[4]. Early work in this field made use of the properties of ultracold and hot atomic gases [2, 3, 5], although more recently interest has turned to room-temperature solid-state systems, which in many ways are better suited for use in practical applications. Solid state slow and fast light systems have been based largely upon the use of coherent population oscillations (CPO) and stimulated light scattering.

In this paper, we analyze some specific aspects of slow light by means of CPO. CPO lead to slow propagation of light for the following reason. If an intense pump field and a detuned signal field simultaneous illuminate a saturable optical material, the ground state population will be decreased as a consequence of saturation effects. This decrease will consist of both a steady state depletion that depends on the average intensity of the applied fields and a time varying contribution that corresponds to the beat frequency between the pump and signal fields. This time varying contribution, that is, the coherent population oscillations, leads to decreased attenuation of the signal field, for reasons that are described mathematically by means of the calculation given below. The decreased absorption occurs only for beat frequencies less than or of the order of the inverse of the ground state population relaxation time $T_{1}$. Thus a narrow transparency window of width $\approx 1 / T_{1}$ will be induced in the absorption profile of the material system. A rapid variation of refractive index will accompany this change in absorption, leading to a large contribution to the group velocity by means of the usual relation $n_{g}=n+\omega d n / d \omega$. Fig- ure 1 shows the CPO hole and associated group and phase indices for parameters valid for ruby.

Slow light by means of CPO has been observed by many workers [4], [6]-[13] with modulation bands ranging from a few $\mathrm{Hz}$ to a few $\mathrm{THz}$. However, some of these results seem paradoxical, in that slow light effects are observed even under conditions such that the line width of the incident laser radiation is much broader than the width of the induced transparency window [16]. For example, in work [6] the effective lifetime for the transition from the ground state to the ${ }^{4} F_{2}$ absorption band in ruby is $4.45 \mathrm{~ms}$, but the linewidth of the $\mathrm{Ar}^{+}$ laser is on the order of $1 \mathrm{GHz}$. The aim of the present study is to show how $\mathrm{CPO}$ effects can occur even under these extreme conditions. We also present an alternative description of slow light effects based on a time domain description that in some ways complements the frequency-domain description.

\section{MATHEMATICAL MODEL}

The propagation of modulated fields through a saturable absorber has been treated in detail in the scientific literature $[14,15]$. Our key point is that slow-light by CPO can be explained entirely within the context of a rate-equation model. This has been noted by other authors [16]; in our work we want to emphasize the fact that the rate-equation model of CPO does predict the slowing down of the group velocity. In a rate equation model, the properties of the laser light enter only in terms of the laser intensity. Thus, the spectral width of the laser light does not directly enter the model at all. Single mode laser light can often by described in a phase diffusion approximation. In this approximation, the laser light is entirely stable in amplitude, and the laser linewidth results purely from phase fluctuations. Thus, in this case, slow light is observed, 
no matter how large the laser line width. However, slow light effects by means of CPO have also been observed in multilongitudinal mode lasers. Such lasers typically display large intensity fluctuations because of beating between different laser modes.

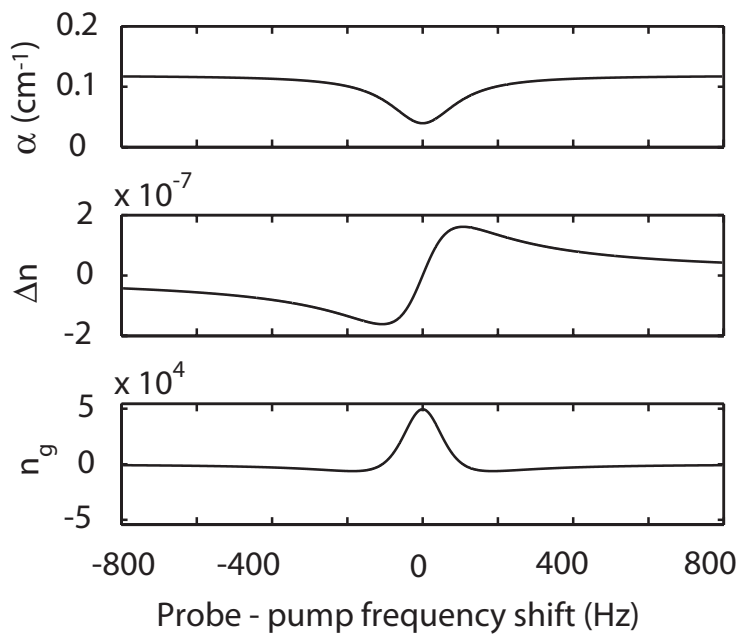

FIG. 1 CPO hole and associated phase and group indices for the ${ }^{4} F_{2}$ absorption band of ruby at a pump intensity equal to twice the saturation intensity; $\alpha$ is the absorption coefficient, $\Delta n=n-1$ the swing in the refractive index and $n_{g}$ the group index.

However, these beat frequencies are often much larger than the inverse of the response time of the material system, and thus the material cannot respond to these frequencies ${ }^{1}$.

Our model is similar to that of one of the early papers on $\mathrm{CPO}$ [17]. We assume that the dipole dephasing time $T_{2}$ of the material system is much smaller than the ground state recovery time $T_{1}$ and the inverse of the Rabi frequency associated with the applied laser fields. In this case, to good approximation we can describe the dynamics of the system through use of a rate equation. We describe the ground state population $N$ by means of the equation

$$
\frac{d N}{d t}=-\frac{N \sigma I_{\text {tot }}(t)}{\hbar \omega}+\frac{(\bar{N}-N)}{T_{1}}
$$

where $\bar{N}$ is the total density of absorbers and $\hbar \omega$ is the energy of the photons of the laser field. The total intensity of the applied field is given by

$$
I_{\text {tot }}(t)=I_{0}+I_{1} e^{-i \Delta t}+\text { c.c. }
$$

Here $\Delta$ is the the modulation frequency of the total intensity. The modulation can occur from the beating of mutually coherent applied fields or can be the result of passing a laser beam through an intensity modulator. The solution of Eq. (1) for an intensity of the form of Eq. (2) is given to lowest order for $I_{1}<<I_{0}$ by

$$
\begin{gathered}
N(t)=\frac{\bar{N}}{1+I_{0} / I_{\text {sat }}}-\left[\frac{\bar{N}}{1+I_{0} / I_{\text {sat }}}\left(\frac{I_{1} / I_{\text {sat }}}{1+I_{0} / I_{\text {sat }}-i \Delta T_{1}}\right) \times\right. \\
\left.e^{-i \Delta t}+\text { c.c. }\right]
\end{gathered}
$$

\footnotetext{
${ }^{1}$ For ruby the effective lifetime for the transition from the ground state to the ${ }^{4} F_{2}$ absorption band of ruby is $4.45 \mathrm{~ms}$ while the line spacing of the $\mathrm{Ar}^{+}$laser used for the experiments is of the order of $100 \mathrm{MHz}$
}

where $I_{\text {sat }}=\hbar \omega / \sigma T_{1}$ is the saturation intensity.

We can now describe the propagation of the optical field according to

$$
\frac{d}{d z} I_{\mathrm{tot}}=-\sigma N(t) I_{\mathrm{tot}}(t)
$$

By extracting from this equation terms with identical time dependences we see that the unmodulated and modulated portions of the field experience different levels of attenuation. In particular, we see that the unmodulated portion obeys the equation

$$
\frac{d}{d z} I_{0}=-\alpha_{0} I_{0}
$$

where

$$
\alpha_{0}=\frac{\bar{N}}{1+I_{0} / I_{\mathrm{sat}}} \sigma
$$

whereas the modulated component obeys

$$
\frac{d}{d z} I_{1}=-\alpha_{1} I_{1}
$$

where

$$
\begin{aligned}
\alpha_{1} & =\alpha_{0}-\sigma \frac{\bar{N}}{1+I_{0} / I_{\mathrm{sat}}}\left(\frac{I_{0} / I_{\mathrm{sat}}}{1+I_{0} / I_{\mathrm{sat}}-i \Delta T_{1}}\right) \\
& =\alpha_{0}-\bar{N} \sigma\left(\frac{I_{0} / I_{\mathrm{sat}}}{1+I_{0} / I_{\mathrm{sat}}}\right)\left(\frac{1+I_{0} / I_{\mathrm{sat}}+i \Delta T_{1}}{\left(1+I_{0} / I_{\mathrm{sat}}\right)^{2}+\left(\Delta T_{1}\right)^{2}}\right)
\end{aligned}
$$

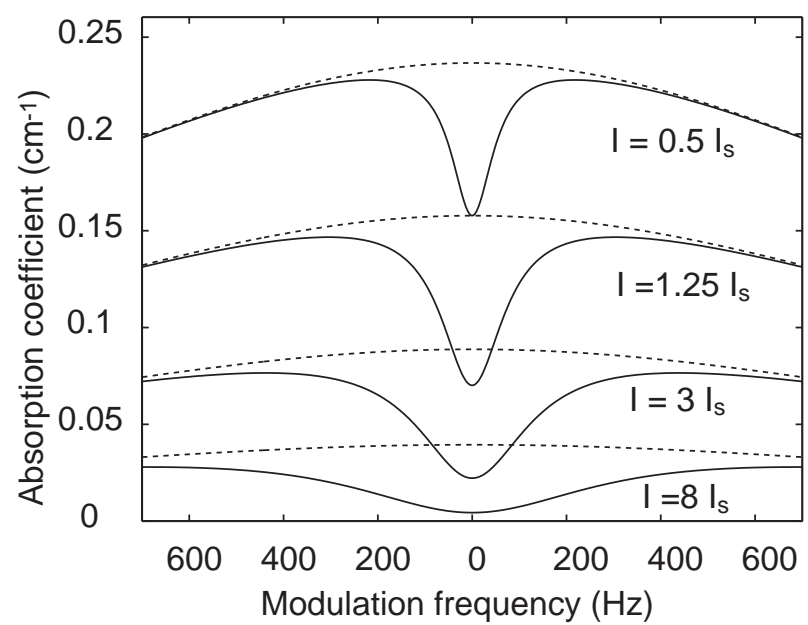

FIG. 2 CPO holes for various values of pump intensity; for phase modulation there is no CPO hole (dashed lines), the CPO hole is present for amplitude modulation (solid lines).

Note that $\alpha_{1}$ is complex. The real part of $\alpha_{1}$, which is plotted for various values of the pump intensity in Figure 2, describes the attenuation of the modulated portion of the laser field, whereas its imaginary part describes a spatially varying change in the phase of the modulation. We can describe spatial variation of the phase of the modulation in terms of a group velocity. In a time interval $T=2 \pi / \Delta$, the modulated waveform will advance a distance $D=2 \pi / \operatorname{Im}\left\{\alpha_{1}\right\}$ (we are considering group velocities so small that we can disregard 
the delay given by propagation at $c$ ). Thus the group velocity $v_{g}=D / T$ will be given by

$$
v_{g}=\frac{\left(1+I_{0} / I_{\mathrm{sat}}\right)\left[\left(1+I_{0} / I_{\mathrm{sat}}\right)^{2}+\left(\Delta T_{1}\right)^{2}\right]}{\bar{N} \sigma T_{1}\left(I_{0} / I_{\mathrm{sat}}\right)}
$$

The predicted group velocity is half that quoted in Ref. [6] which uses a density matrix treatment. The current model automatically includes four-wave mixing contributions that were excluded from the earlier calculation.

\section{NON-COLLINEAR GEOMETRY}

Absorption of the pump beam limits the maximum delay that can be obtained with the CPO technique. The pump decays more quickly than the probe because it feels only the effect of saturated absorption; as the pump intensity decays the CPO hole and the associated slowing down of the probe disappear. A possible solution to this problem is to replenish the pump; this, for example, could be done easily if the direction of propagation of the pump and probe are different. Two possible such geometries are shown in Figure 3. We next demonstrate that CPO lead to slow light also when pump and probe are propagating in different directions. The effect can be described by applying the density matrix formalism to a two-level atom; we modify the treatment of [18] to obtain the phase and group velocity of the probe. (a)

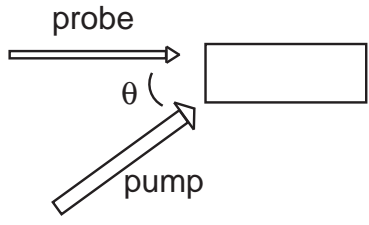

(b)

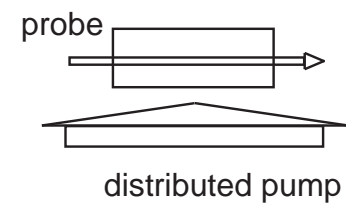

FIG. 3 (a) non-collinear CPO; (b) distributed pump.

The two-level atom is described by a density matrix $\rho_{i j} ;|a\rangle$ is the lower level and $|b\rangle$ is the upper level. The dipole matrix element is $\mu_{b a}=\mu_{a b}^{*}=\langle b|-e x| a\rangle, T_{1}$ is the population lifetime and $T_{2}$ is the dipole dephasing lifetime; $N$ indicates the number density of atoms. The pump and probe fields are denoted respectively as

$$
\tilde{E}(\overrightarrow{\mathbf{r}}, t)=E_{j} e^{-i \omega_{j} t}+E_{j}^{*} e^{i \omega_{j} t}
$$

where

$$
E_{j}=A_{j} e^{i \overrightarrow{\mathbf{k}}_{j} \cdot \overrightarrow{\mathbf{r}}}
$$

and $j$ is equal to pump and probe respectively for the pump and probe fields. We assume that the pump field is tuned to the atomic resonance $\omega_{b a}$. The frequency difference between the two fields is $\delta=\omega_{\text {probe }}-\omega_{\text {pump }}$.

When the dephasing width $T_{2}^{-1}$ is large with respect to both the Rabi frequency and the beat frequency $\delta$ the diagonal term of the density matrix can be expressed as a function of the inversion as

$$
\begin{gathered}
\rho_{b a}(t) \approx-i \frac{\mu_{b a} T_{2}}{\hbar}\left(E_{\text {pump }} e^{-i \omega_{\text {pump }} t}+E_{\text {probe }} e^{-i \omega_{\text {probe }} t}\right) \times \\
{\left[\rho_{b b}(t)-\rho_{a a}(t)\right]}
\end{gathered}
$$

This allows one to obtain a simple formula for the population inversion, which can be substituted back into Eq. (12) to obtain the polarization. We write the inversion as in ref. [18], but we retain the phase difference between the pump and probe waves that comes from their different spatial dependence. It is

$$
\begin{aligned}
\rho_{b b}-\rho_{a a} \simeq\left(\rho_{b b}-\rho_{a a}\right)^{(d c)} & +\left(\rho_{b b}-\rho_{a a}\right)^{(\delta)} e^{i \delta t} \\
& +\left(\rho_{b b}-\rho_{a a}\right)^{(-\delta)} e^{-i \delta t}
\end{aligned}
$$

with

$$
\begin{gathered}
\left(\rho_{b b}-\rho_{a a}\right)^{(d c)}=\frac{\left(\rho_{b b}-\rho_{a a}\right)^{(0)}}{\beta T_{1}} \\
\left(\rho_{b b}-\rho_{a a}\right)^{(\delta)}=-\frac{\left(\rho_{b b}-\rho_{a a}\right)^{(0)}}{T_{1}} \gamma \frac{1-i \delta / \beta}{\delta^{2}+\beta^{2}} \\
\left(\rho_{b b}-\rho_{a a}\right)^{(-\delta)}=-\frac{\left(\rho_{b b}-\rho_{a a}\right)^{(0)}}{T_{1}} \gamma^{*} \frac{1+i \delta / \beta}{\delta^{2}+\beta^{2}}
\end{gathered}
$$

The quantities $\beta$ and $\gamma$ are given by

$$
\begin{aligned}
& \beta=\frac{1}{T_{1}}+\frac{4 T_{2}\left|\mu_{b a}\right|^{2}}{\hbar^{2}}\left|E_{\text {pump }}\right|^{2} \\
& \gamma=\frac{4 T_{2}\left|\mu_{b a}\right|^{2}}{\hbar^{2}} E_{\text {pump }} E_{\text {probe }}^{*}
\end{aligned}
$$

$\left(\rho_{b b}-\rho_{a a}\right)^{(0)}$ is the equilibrium value of the inversion.

The polarization of the two-level atom medium, $P=N \rho_{b a} \mu_{a b}$ contains a term that oscillates at $\omega_{\text {pump }}$ and a term that oscillates at $\omega_{\text {probe }}$ :

$$
P\left(\omega_{\text {pump }}\right)=\frac{-i N\left|\mu_{a b}\right|^{2} T_{2}}{\hbar} \frac{\left(\rho_{b b}-\rho_{a a}\right)^{(0)}}{1+\frac{4}{\hbar^{2}}\left|\mu_{a b}\right|^{2}\left|E_{\text {pump }}\right|^{2} T_{1} T_{2}} E_{\text {pump }}
$$

$$
\begin{aligned}
P\left(\omega_{\text {probe }}\right) & =\frac{-i N\left|\mu_{a b}\right|^{2} T_{2}}{\hbar}\left(\rho_{b b}-\rho_{a a}\right)^{(0)} \times \\
& {\left[\frac{1}{1+\frac{4}{\hbar^{2}}\left|\mu_{a b}\right|^{2}\left|E_{\text {pump }}\right|^{2} T_{1} T_{2}}-\right.} \\
& \left.\frac{4 T_{2}\left|\mu_{b a}\right|^{2}}{\hbar^{2} T_{1}}\left|E_{\text {pump }}\right|^{2} \frac{1+i \delta / \beta}{\delta^{2}+\beta^{2}}\right] E_{\text {probe }}
\end{aligned}
$$

where we have retained only terms that are first order in $E_{\text {probe }}$. The polarization given by Eqs. (19) and (20) can be substituted in the wave equation

$$
-\nabla^{2} E+\frac{1}{c^{2}} \frac{\partial^{2} E}{\partial t^{2}}=-\frac{4 \pi}{c^{2}} \frac{\partial^{2} P}{\partial t^{2}}
$$

The wave equation contains components of the polarization that are phase matched with $E_{\text {pump }}$ and $E_{\text {probe }}$ respectively and which are functions of the pump wave intensity; $E_{\text {pump }}$ and $E_{\text {probe }}$ vary slowly along their respective direction of propagation because of these polarization components. It is possible to derive separate equations for $E_{\text {pump }}$ and $E_{\text {probe, }}$ as described for example in section 7.4 of ref [19]. The equation for $E_{\text {pump }}$ shows saturable absorption, while the equation for $E_{\text {probe }}$ is 


$$
\begin{aligned}
& \frac{\partial E_{\text {probe }}}{\partial z}=\frac{2 \pi \omega}{c}\left(\frac{N\left|\mu_{b a}\right|^{2} T_{2}}{\hbar}\right)\left(\rho_{b b}-\rho_{a a}\right)^{(0)} \times \\
& {\left[\frac{1}{1+\frac{4}{\hbar^{2}}\left|\mu_{a b}\right|^{2}\left|E_{\text {pump }}\right|^{2} T_{1} T_{2}}-\right.} \\
&\left.\frac{4 T_{2}\left|\mu_{b a}\right|^{2}}{\hbar^{2} T_{1}}\left|E_{\text {pump }}\right|^{2} \frac{1+i \delta / \beta}{\delta^{2}+\beta^{2}}\right] E_{\text {probe }}
\end{aligned}
$$

In Eq. (22) we recognize the saturation of the absorption due to the pump wave and the population oscillations. The phase delay due to population oscillations leads to a group velocity for a probe wave whose intensity is modulated at a frequency $2 \delta$ given, in the approximation $v_{g}<<c$, by

$$
v_{g}=\frac{2\left(1+I_{\text {pump }} / I_{\text {sat }}\right)\left[\left(1+I_{\text {pump }} / I_{\text {sat }}\right)^{2}+\left(\delta T_{1}\right)^{2}\right]}{N \sigma T_{1}\left(I_{\text {pump }} / I_{\text {sat }}\right)}
$$

In order to obtain expression (23) we have recognized the cross section $\sigma=(4 \pi \omega / c)\left(\left|\mu_{b a}\right|^{2} T_{2} / \hbar\right)$ and the ratio between the pump intensity and saturation intensity $I_{\text {pump }} / I_{\text {sat }}=\left(4\left|\mu_{b a}\right|^{2}\left|E_{\text {pump }}\right|^{2} / \hbar^{2}\right) T_{1} T_{2}$ in Eq. (22) and we have set $\left(\rho_{\mathrm{bb}}-\rho_{\mathrm{aa}}\right)^{(0)}=-1$.

The velocity at which a modulation propagates in the case of non-collinear propagation is different from the collinear propagation case because the contribution of the four-wave mixing terms is absent for non-collinear propagation.

\section{S U M M A Y A N D C N CLUSIONS}

In summary we have shown, using a rate equations model that the CPO hole can be created in a saturable absorber even when the bandwidth of the pump and probe beams is much larger than the inverse of the response time of the material. We have shown moreover that CPO can slow down light also when pump and probe propagate in different directions; the non-collinear configuration can be useful for removing the limitation to the maximum delay achievable with CPO given by the absorption of the pump.

This work was supported by the DARPA/DSO Slow Light Program and by the NSF.

\section{References}

[1] R. W. Boyd and D. J. Gauthier, "“Slow" and "fast" light", Progress In Optics, Volume 4343 497-530 (2002).

[2] L. V. Hau, S. E. Harris, Z. Dutton, and C. H. Behroozi, "Light speed reduction to 17 metres per second in an ultracold atomic gas" Nature 397, 594-598 (1999).

[3] L. Wang, A. Kuzmich, and A. Dogariu, "Cain-assisted superluminal light propagation" Nature 406, 277-279 (2000).
[4] M. S. Bigelow, N. N. Lepeshkin, and R. W. Boyd, "Superluminal and slow light propagation in a room-temperature solid" Science 301, 200-202 (2003).

[5] M. M. Kash, V. A. Sautenkov, A. S. Zibrov, L. Hollberg, G. R. Welch, M. D. Lukin, Y. Rostovtsev, E. S. Fry, and M. 0. Scully, "Ultraslow group velocity and enhanced nonlinear optical effects in a coherently driven hot atomic gas" Physical Review Letters 82, 5229-5232 (1999).

[6] M. S. Bigelow, N. N. Lepeshkin, and R. W. Boyd, "Observation of UItraslow Light Propagation in a Ruby Crystal at Room Temperature" Physical Review Letters 90, 113903 (2003).

[7] P.-C. Ku, F. Sedgwick, C. J. Chang-Hasnain, P. Palinginis, T. Li, H. Wang, S.-W. Chang, and S.-L. Chuang, "Slow light in semiconductor quantum wells" Optics Letters 29, 2291-2293 (2004).

[8] P. Palinginis, F. Sedgwick, S. Crankshaw, M. Moewe, and J. C. Chang-Hasnain, "Room temperature slow light in a quantum-well waveguide via coherent population oscillation" Optics Express 13, 9909-9915 (2005).

[9] E. Baldit, K. Bencheikh, P. Monnier, J. Levenson, and V. Rouget, "Ultraslow Light Propagation in an Inhomogeneously Broadened Rare-Earth Ion-Doped Crystal" Physical Review Letters 95, 143601 (2005).

[10] P. Wu and D. V. G. L. N. Rao, "Controllable Snail-Paced Light in Biological Bacteriorhodopsin Thin Film" Physical Review Letters 95, 253601 (2005).

[11] M. van der Poel, J. Moerk, and J. M. Hvam, "Controllable delay of ultrashort pulses in a quantum dot optical amplifier" Optics Express 13, 8032-8037 (2005).

[12] J. Moerk, R. Kjaer, M. van der Poel, and K. Yvind, "Slow light in a semiconductor waveguide at gigahertz frequencies" Optics Express $13,8136-8145$ (2005).

[13] H. Su and S. L. Chuang, "Room-temperature slow light with semiconductor quantum-dot devices" Optics Letters 31, 271-273 (2006).

[14] L. W. Hillman, R. W. Boyd, and J. C. R. Stroud, "Natural modes for the analysis of optical bistability and laser instability" Optics Letters 7, 426 (1982).

[15] M. A. Kramer, R. W. Boyd, L. W. Hillman, and J. C. R. Stroud, "The propagation of modulated optical fields through saturable absorbing media: a general theory of modulation spectroscopy" Journal of the Optical Society of America B 2, 1444 (1985).

[16] V. S. Zapasskii and G. G. Kozlov, "A saturable absorber, coherent population oscillations and slow light" Optics and Spectroscopy 100, 419-424 (2006).

[17] M. S. Malcuit, R. W. Boyd, L. W. Hillmann, J. Krasinski, and J. C. R. Stroud, "Saturation and inverse-saturation absorption line shapes in alexandrite" Journal of the Optical Society of America B 1, 73-75 (1984).

[18] R. W. Boyd, M. G. Raymer, P. Narum, and D. J. Harter, "Fourwave parametric interaction in a strongly driven two-level system" Physical Review A 24, 411-423 (1981).

[19] R. W. Boyd, Nonlinear Optics (second edn., pp. 350-355 Academic Press, 2003). 